\title{
Toward Behavioral Benchmarks for Whisker-Related Sensory Processing
}

\author{
Maik C. Stüttgen \\ Department of Biopsychology, Institute of Cognitive Neuroscience, Faculty of Psychology, University of Bochum, D-44780 Bochum, Germany \\ Review of O'Connor et al.
}

The rodent whisker-to-barrel pathway serves as a major model system for the investigation of sensorimotor processing. Its key advantage is a well defined topographic map, in which each of the orderly arranged whiskers on the snout of the animal is processed largely in a corresponding functional column in primary somatosensory ("barrel") cortex. This topographic map is situated on top of a lissencephalic cortex, and is thus easily accessible for electrodes or imaging microscopes. The map can be easily altered, e.g., by plucking whiskers during different stages of brain development, allowing study of plasticity mechanisms. Furthermore, rodents use their whiskers for exploration in a highly active manner, rhythmically sweeping them back and forth ("whisking"), which provides the opportunity to study sensorimotor integration in a system with considerably fewer degrees of freedom than primate hands.

Although barrel cortex is well described in terms of microcircuitry (Lübke and Feldmeyer, 2007) and mechanisms of plasticity (Feldman and Brecht, 2005), there is a paucity of behavioral data on whisker usage. While rats clearly can use their whiskers for object localization (Hutson and Masterton, 1986) and texture discrimination (Carvell and Simons, 1990), little is known about

Received Feb. 9, 2010; revised March 4, 2010; accepted March 8, 2010.

Correspondence should be addressed to Maik C. Stüttgen, Department of Biopsychology, Institute of Cognitive Neuroscience, Faculty of Psychology, GAFO 05/620, University of Bochum, D-44780 Bochum, Germany. E-mail:maik.stuettgen@rub.de.

DOI:10.1523/JNEUROSCI.0713-10.2010

Copyright $\odot 2010$ the authors $\quad 0270-6474 / 10 / 304827-03 \$ 15.00 / 0$ how the animals employ their whiskers to accomplish these tasks. This situation is largely because of a lack of methods that allow precise quantification of whisker movements while, at the same time, permitting psychophysical assessments. The intricate interplay of whiskers and surfaces during free exploration calls for analysis with millisecond resolution and micrometer precision and can most accurately be examined using high-speed videography, which in turn requires sophisticated analysis software to track single whiskers (Ritt et al., 2008).

Because of the lack of psychophysical whisker-based paradigms and the difficulties in measuring whisking, experiments and theories on whisker sensory processing have been largely unconstrained by behavioral benchmarks. For example, investigators have routinely stimulated whiskers with amplitudes and velocities that have recently been found to be on the lower end of the spectrum rats encounter during exploration (Ritt et al., 2008) and, in fact, are occasionally close to absolute perceptual threshold (Stüttgen et al., 2006). Similarly, some studies have presented stimuli (e.g., surfaces of differing roughness like sandpapers) to the whiskers that have not been shown to be discriminable by trained animals.

In a similar vein, a recent study compared the information contents of spike count versus the spikes' temporal pattern for whisker-based texture discrimination in anesthetized animals (Arabzadeh et al., 2006), and found that analysis of spike patterns allows better discriminability of tex- tures than analysis of spike counts. This finding may be taken to suggest that the rat does indeed base its capacity for texture discrimination on spike patterns, but is not conclusive of the coding symbol that is used. Also, it is not known how well trained animals can distinguish between the stimuli these authors presented. However, combining neural recordings with a behavioral task may solve this issue, as has been done in the primate tactile system. Hernández et al. (2000) found that, similar to the whisker system, the temporal patterns of spike trains (periodicity) in primary somatosensory cortex provided more information about the frequency of a vibrotactile signal than spike counts, thus permitting superior discrimination performance. However, neurometric discriminability based on periodicity by far exceeded the discriminability shown by the animals, while neurometric discriminability based on spike counts yielded a good match to the psychometric data. This result and additional comparisons of behavioral and neuronal data led to the conclusion that the neural code for frequency discrimination is spike count rather than spike periodicity (Luna et al., 2005).

In summary, research on neural processing in the whisker system needs to be linked to behavioral benchmarks: which surfaces and objects can be discriminated, and how well? What forces impinge on whiskers during object localization or texture discrimination? What strategies do animals apply to meet different task demands, and how do these strategies affect neural processing at different levels of the whisker-to-barrel 
a

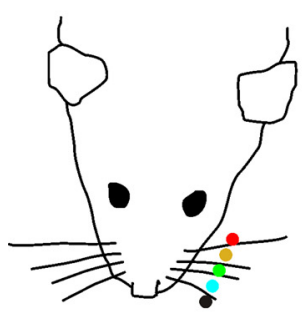

b

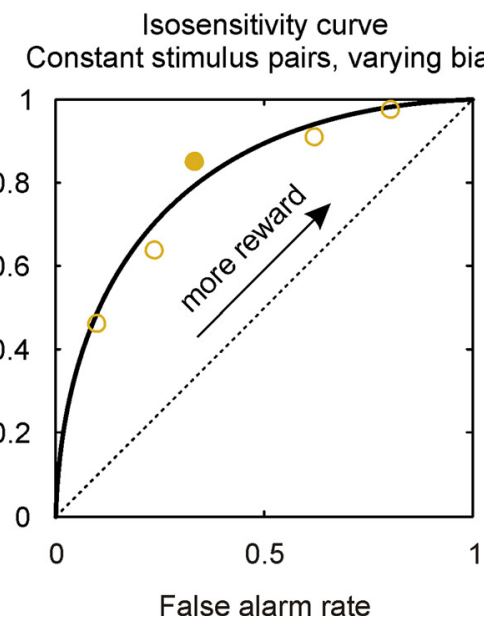

C

Isobias curve

Varying stimulus pairs, constant bias

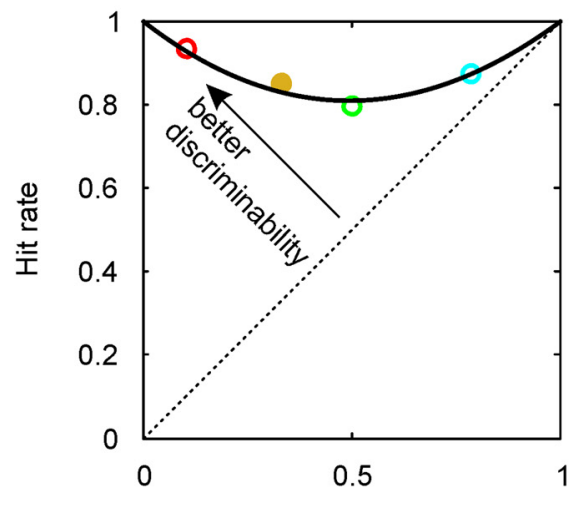

False alarm rate

Figure 1. Proposed signal detection theoretical analysis of the go/no-go task. $\boldsymbol{a}$, Top view of a mouse head with five different stimuli. In each session, a no-go stimulus and one of four go stimuli (rods at different positions) are presented in random order. The animal should respond only when a go stimulus is present. The smaller the distance between go and no-go stimuli, the more difficult the task. $\boldsymbol{b}$, Idealized isosensitivity curve for one stimulus pair, yellow rod versus no go. Each data point is obtained from a session with the same stimulus pair, but the amount of reward for a correct (hit) response is varied across sessions. SDT predicts that both hit and false alarm rates increase together with reward amount, and data points should lie on the bold curve. $\boldsymbol{c}$, Idealized isobias curve for four stimulus pairs (four colored rods vs no-go; see $\boldsymbol{a}$ ). Each data point is derived from a session with different stimulus pairs, but the amount of reward for a correct response was held constant across sessions. SDT predicts that data points should fall close to the bold line if the subject compares the likelihoods of go and no-go stimuli on each trial.

pathway? Ideally, such behavioral assessments should be conducted with simultaneous recording of neural activity.

One way to combine precise whisker tracking and psychophysical assessment is to use head-fixed animals performing whisker-based discrimination tasks (Harvey et al., 2001). Therefore, a recently introduced adaptation of the head-fixed behaving primate preparation to mice by O'Connor et al. (2010) comes as a welcome addition. The authors implanted mice with head posts for fixation and trained them on a whisker-based object localization task. They used a go/no-go task in which mice were rewarded for licking from a water spout when a metal rod was present at a target position relative to the animal's snout, but punished for licking when the rod was in another (distracter) position. Mice showed evidence of discriminating between go and no-go stimuli early in training, within as few as 7-14 sessions. Moreover, mice generalized their knowledge of task requirements with easy stimuli to more difficult stimulus sets.

In addition to introducing a novel behavioral task for mice, the authors reported interesting findings regarding the discrimination behavior. For example, mice could perform the location task using only a single whisker. Also, contralateral but not ipsilateral barrel cortex was necessary for performance of the discrimination task, as assessed by temporary inactivation of these structures by local infusion of the GABA agonist muscimol. These findings are consistent with previous work in unrestrained rats (Hutson and Masterton, 1986; Mehta et al.,
2007) and demonstrate the usefulness and validity of the paradigm.

The head-fixed mouse preparation is well suited to investigations of task-related neural activity in combination with psychophysical assessments. First, mice performed hundreds of trials in sequence, which is important when sampling signals with a high trial-to-trial variability such as action potential responses. Second, the large number of trials was achieved in a time span of typically less than $1 \mathrm{~h}$, which is important for recording single-unit activity extracellularly or intracellularly because of limited recording durations. Thirdly, animals learned the task quickly, requiring 7-14 sessions to reach a performance criterion of $85 \%$ correct responses. However, this time span should probably be viewed as a lower bound needed for training. Performance on psychophysical tasks typically increases over a period of several weeks, especially when novel stimuli are continuously added. For researchers aiming to tackle absolute psychophysical threshold and to relate performance to neural responses (e.g., Britten et al., 1992), extended training will be required to work animals down to stable asymptotic thresholds and to reduce within-session performance fluctuations. Still, visual inspection of the mice's learning curves [O'Connor et al. (2010), their Fig. 3] indicates that overtraining to easy stimuli can take as little as five more sessions, and within-session fluctuations in performance decrease markedly.

While O'Connor et al. (2010) deserve credit for their achievements, there is some room for improvement regarding their psychophysical measurements. The authors refrained from constructing full psychometric curves within a single session because they reasoned that mice would respond only in easy trials and ignore near-threshold stimuli. Previous research in head-fixed rats suggests that this concern is unwarranted; in another psychophysical study, animals were tested successfully with up to 10 nearthreshold whisker stimuli per session (Stüttgen and Schwarz, 2008). Clearly, psychophysical testing with more than two stimuli is desirable for investigating the detailed quantitative relationship between neural activity and the animals' percepts (Britten et al., 1992).

At the same time, the measurement of a full psychometric curve within one session may solve a problem the authors encountered. When confronted with more difficult discriminations, the mice displayed a large fraction of false alarms [O'Connor et al. (2010), their Fig. 7b]. This makes it difficult to disentangle true hit responses (i.e., those based on a correct perceptual judgment) from those hits achieved by a pure guessing strategy (i.e., random licking). In the extreme case, mice displayed false alarm rates approaching 1 . This suggests that the reinforcing effects of water delivery for hits considerably outmatched the punishing effects of the airpuff for false alarms.

However, this shortcoming could also be turned into an advantage: studying the effects of varying reward amount for hits to construct isosensitivity curves within the framework of signal detection theory (SDT) 
(Green and Swets, 1966). Such curves are obtained by presenting the same stimulus pair (in this case, one no-go stimulus with any one of several go stimuli) (Fig. 1a) over and over again, while varying the amount of reward for correct and incorrect responses, respectively. In this scenario, subjects typically adjust the relative probabilities of hits and false alarms to maximize reward ("response bias"), and SDT predicts that hits and false alarms bear a strict quantitative relationship to each other (see Fig. $1 b$ for an example). Moreover, the data of mouse JF 4793 and especially JF3465 roughly conform to another postulate of SDT: the shape of isobias curves. Complementary to isosensitivity curves, isobias curves are collected by presenting stimulus pairs of differing discriminability but constant reward amount for correct responses [see Fig. $1 c$ for an example; compare to O'Connor et al. (2010), their Fig. 7b]. While the authors did not conduct a systematic assessment of the shapes of isosensitivity and isobias curves, the data of two of the four mice resemble those frequently found in signal detection tasks. This result lends further credibility to the results and opens up the possibility of studying neural correlates of perceptual decision making in mice performing this discrimination task, and linking neural data to a well established psychological theory of decision making.

In the past decades, the gold standard for the investigation of the relationship between brain and behavior has been the head-fixed awake behaving primate preparation (Evarts, 1966). This preparation offers unique stimulus control in terms of highly precise stimulus application and response recording, while at the same time providing great opportunities for recording neural activity. This approach has provided a host of novel insights into processes of sensation and perception (Newsome et al., 1989), decision making (Platt and Glimcher, 1999), and motor control (Georgopoulos et al., 1986). Advantages of the whisker system over primate sensory systems are detailed knowledge of microcircuits, mechanisms of plasticity, and barrel cortex's accessibility for optical imaging of neural activity. The wide availability of transgenic mouse lines, in combination with behavioral assays as described by O'Connor et al. (2010), hold great promise for novel insights regarding the relationship of brain and behavior.

\section{References}

Arabzadeh E, Panzeri S, Diamond ME (2006) Deciphering the spike train of a sensory neuron: counts and temporal patterns in the rat whisker pathway. J Neurosci 26:9216-9226.

Britten KH, Shadlen MN, Newsome WT, Movshon JA (1992) The analysis of visual motion: a comparison of neuronal and psychophysical performance. J Neurosci 12:4745-4765.

Carvell GE, Simons DJ (1990) Biometric analyses of vibrissal tactile discrimination in the rat. J Neurosci 10:2638-2648.

Evarts EV (1966) Methods for recording activity of individual neurons in moving animals. In: Methods in medical research (Rushmer RF, ed), pp 241-250. Chicago: Year Book.

Feldman DE, Brecht M (2005) Map plasticity in somatosensory cortex. Science 310:810-815.

Georgopoulos AP, Schwartz AB, Kettner RE (1986) Neuronal population coding of movement direction. Science 233:1416-1419.

Green DM, Swets JA (1966) Signal detection theory and psychophysics. New York: Wiley.

Harvey MA, Bermejo R, Zeigler HP (2001) Dis- criminative whisking in the head-fixed rat: optoelectronic monitoring during tactile detection and discrimination tasks. Somatosens Mot Res 18:211-222.

Hernández A, Zainos A, Romo R (2000) Neuronal correlates of sensory discrimination in the somatosensory cortex. Proc Natl Acad Sci U S A 97:6191-6196.

Hutson KA, Masterton RB (1986) The sensory contribution of a single vibrissa's cortical barrel. J Neurophysiol 56:1196-1223.

Lübke J, Feldmeyer D (2007) Excitatory signal flow and connectivity in a cortical column: focus on barrel cortex. Brain Struct Funct 212:3-17.

Luna R, Hernández A, Brody $\mathrm{CD}$, Romo R (2005) Neural codes for perceptual discrimination in primary somatosensory cortex. Nat Neurosci 8:1210-1219.

Mehta SB, Whitmer D, Figueroa R, Williams BA, Kleinfeld D (2007) Active spatial perception in the vibrissa scanning sensorimotor system. PLoS Biol 5:e15.

Newsome WT, Britten KH, Movshon JA (1989) Neuronal correlates of a perceptual decision. Nature 341:52-54.

O'Connor DH, Clack NG, Huber D, Komiyama T, Myers EW, Svoboda K (2010) Vibrissabased object localization in head-fixed mice. J Neurosci 30:1947-1967.

Platt ML, Glimcher PW (1999) Neural correlates of decision variables in parietal cortex. Nature 400:233-238.

Ritt JT, Andermann ML, Moore CI (2008) Embodied information processing: vibrissa mechanics and texture features shape micromotions in actively sensing rats. Neuron 57:599-613.

Stüttgen MC, Schwarz C (2008) Psychophysical and neurometric detection performance under stimulus uncertainty. Nat Neurosci 11: 1091-1099.

Stüttgen MC, Rüter J, Schwarz C (2006) Two psychophysical channels of whisker deflection in rats align with two neuronal classes of primary afferents. J Neurosci 26:7933-7941. 